\title{
Local Non-Bayesian Social Learning with Stubborn Agents
}

\author{
DANIEL VIAL, University of Michigan, USA \\ VIJAY SUBRAMANIAN, University of Michigan, USA
}

\begin{abstract}
ACM Reference Format:
Daniel Vial and Vijay Subramanian. 2019. Local Non-Bayesian Social Learning with Stubborn Agents. In ACM EC '19: ACM Conference on Economics and Computation (EC '19), fune 24-28, 2019, Phoenix, AZ, USA. ACM, New York, NY, USA, 2 pages. https://doi.org/10.1145/3328526.3329572
\end{abstract}

In recent years, people have increasingly turned to social networks like Twitter and Facebook for news. In contrast to traditional news sources, these platforms allow users to simultaneously read articles and share opinions with other users. Among other effects, this has led to the rise of fake news, sometimes spread via bots (automated social media accounts masquerading as real users).

In this paper, we study a mathematical model describing this situation. The model includes a large number of agents attempting to learn an underlying true state of the world (e.g. which of two candidates is better suited for office) using information from three sources. First, each agent receives noisy observations of the true state, modeling e.g. news stories from major publications. Second, each agent observes the opinions of a subset of other agents, modeling e.g. discussions with other social media users. Third, each agent may observe the opinions of stubborn agents or bots who aim to persuade others of an erroneous true state, modeling e.g. users spreading fake news. Based on this information, agents iteratively update their beliefs about the true state in a manner similar to the non-Bayesian social learning model of Jadbabaie et al. [5]. This iterative process continues for a finite number of iterations that we refer to as the learning horizon.

Under this model, two competing forces emerge as the learning horizon grows. On the one hand, agents receive more observations of the true state, suggesting that they become more likely to learn. On the other hand, the influence of bots gradually propagates through the system, suggesting that agents become increasingly susceptible to this influence and thus less likely to learn. Hence, while the horizon clearly affects the learning outcome, the nature of this effect is less clear.

This effect of the learning horizon has often been ignored in works with models similar to ours. For example, our model is nearly identical to that in the empirical work [2], in which the authors show that polarized beliefs can arise when there are two types of bots with diametrically opposed viewpoints. However, the experiments in [2] simply fix a large learning horizon and do not consider the effect of varying it. Models similar to ours have also been treated analytically; for example, $[1,4,5]$ study non-Bayesian learning models similar to ours. However, these works consider a fixed number of agents and an infinite learning horizon and thus also ignore timescale effects.

The main message of this work is that the learning horizon plays a prominent role in the learning outcome and therefore should not be ignored. In particular, we show that the learning outcome depends on the relationship between the horizon $T_{n}$ and a quantity $p_{n}$ that describes the number of bots in the system, where both quantities may depend on the number of agents $n$. Mathematically,

Permission to make digital or hard copies of part or all of this work for personal or classroom use is granted without fee provided that copies are not made or distributed for profit or commercial advantage and that copies bear this notice and the full citation on the first page. Copyrights for third-party components of this work must be honored. For all other uses, contact the owner/author(s).

EC '19, fune 24-28, 2019, Phoenix, AZ, USA

(C) 2019 Copyright held by the owner/author(s).

ACM ISBN 978-1-4503-6792-9/19/06.

https://doi.org/10.1145/3328526.3329572 
letting $\theta \in(0,1)$ denote the true state and $\theta_{T_{n}}\left(i^{*}\right)$ denote the belief about the true state for a uniformly random agent $i^{*}$ at the horizon $T_{n}$, we show

$$
\theta_{T_{n}}\left(i^{*}\right) \underset{n \rightarrow \infty}{\stackrel{\mathbb{P}}{\longrightarrow}}\left\{\begin{array}{ll}
\theta, & T_{n}\left(1-p_{n}\right) \underset{n \rightarrow \infty}{\longrightarrow} 0 \\
0, & T_{n}\left(1-p_{n}\right) \underset{n \rightarrow \infty}{\longrightarrow} \infty
\end{array},\right.
$$

where $\stackrel{\mathbb{P}}{\rightarrow}$ denotes convergence in probability. Here $p_{n}$ is smaller when more bots are present and 0 is the erroneous true state promoted by the bots. Hence, in words, (1) says the following: if there are few bots, in the sense that $T_{n}\left(1-p_{n}\right) \rightarrow 0, i^{*}$ learns the true state; if there are many bots, in the sense that $T_{n}\left(1-p_{n}\right) \rightarrow \infty, i^{*}$ adopts the extreme belief 0 promoted by the bots. An interesting consequence arises from taking $T_{n, 1}=o\left(1 /\left(1-p_{n}\right)\right)$ and $T_{n, 2}=\omega\left(1 /\left(1-p_{n}\right)\right)$, so that

$$
\theta_{T_{n, 1}}\left(i^{*}\right) \underset{n \rightarrow \infty}{\stackrel{\mathbb{P}}{\longrightarrow}} \theta, \quad \theta_{T_{n, 2}}\left(i^{*}\right) \underset{n \rightarrow \infty}{\stackrel{\mathbb{P}}{\longrightarrow}} 0 .
$$

In words, a typical agent initially (at time $T_{n, 1}$ ) learns the true state $\theta$, then later (at time $T_{n, 2}$ ) "forgets" the true state and adopts the extreme opinion 0.

In addition to (1), we prove two other results. First, we consider a regime that falls between the two in (1); namely, the case $T_{n}\left(1-p_{n}\right) \rightarrow c \in(0, \infty)$ (note larger $c$ implies more bots). In this case, the learning outcome depends on another parameter, which we denote by $\eta \in(0,1)$ and which dictates the weight agents place on other agents' opinions in their belief updates. Here we show

$$
\theta_{T_{n}}\left(i^{*}\right) \underset{n \rightarrow \infty}{\stackrel{\mathbb{P}}{\longrightarrow}} \theta\left(1-e^{-c \eta}\right) /(c \eta) .
$$

The limit in (2) is a convex, decreasing function of $c$, which offers an intuitive explanation: if an adversary deploys bots in hopes of driving agent opinions to 0 , the marginal benefit of deploying additional bots is smaller when more bots have been deployed, i.e. the adversary experiences diminishing returns. Second, we show all but $o(n)$ agents adopt opinion 0 in a certain sub-case of $T_{n}\left(1-p_{n}\right) \rightarrow \infty$; we also argue empirically that this stronger result likely fails in other cases.

Finally, we note our results assume the graph connecting agents and bots follows the directed configuration model, a well-studied random graph model [3]. We also assume $T_{n}=O(\log n)$. Together, these assumptions guarantee that, at the learning horizon, an agent's belief is only influenced by its local neighborhood in the graph (as alluded to by the title of this work).

For the full paper, see [6].

\section{REFERENCES}

[1] Daron Acemoglu, Asuman Ozdaglar, and Ali ParandehGheibi. 2010. Spread of (mis) information in social networks. Games and Economic Behavior 70, 2 (2010), 194-227.

[2] Marina Azzimonti and Marcos Fernandes. 2018. Social media networks, fake news, and polarization. Technical Report. National Bureau of Economic Research.

[3] Ningyuan Chen and Mariana Olvera-Cravioto. 2013. Directed random graphs with given degree distributions. Stochastic Systems 3, 1 (2013), 147-186.

[4] Benjamin Golub and Matthew O Jackson. 2010. Naive learning in social networks and the wisdom of crowds. American Economic fournal: Microeconomics 2, 1 (2010), 112-49.

[5] Ali Jadbabaie, Pooya Molavi, Alvaro Sandroni, and Alireza Tahbaz-Salehi. 2012. Non-Bayesian social learning. Games and Economic Behavior 76, 1 (2012), 210-225.

[6] Daniel Vial and Vijay Subramanian. 2019. Local non-Bayesian social learning with stubborn agents. arXiv preprint arXiv:1904.12767 (2019). 